\title{
A new case of GABA transaminase deficiency facilitated by proton MR spectroscopy
}

\author{
Megumi Tsuji • Noriko Aida • Takayuki Obata Moyoko Tomiyasu • \\ Noritaka Furuya • Kenji Kurosawa • Abdellatif Errami • K. Michael Gibson • \\ Gajja S. Salomons • Cornelis Jakobs • Hitoshi Osaka
}

Received: 2 October 2009 /Revised: 29 October 2009 / Accepted: 23 November 2009 / Published online: 6 January 2010

(C) The Author(s) 2009. This article is published with open access at Springerlink.com

\begin{abstract}
Background Deficiency of 4-aminobutyrate aminotransferase (GABA-T) is a rare disorder of GABA catabolism, with only a single sibship reported. We report on a third case, a Japanese female infant with severe psychomotor retardation and recurrent episodic lethargy with intractable seizures, with the diagnosis facilitated by proton magnetic resonance (MR) spectroscopy $\left({ }^{1} \mathrm{H}-\mathrm{MRS}\right)$.

Methods Neuroimaging was performed at the first episode of lethargy. For ${ }^{1} \mathrm{H}-\mathrm{MRS}$, locations were placed in the semioval center and the basal ganglia. Quantification of metabolite concentrations were derived using the LCModel. We confirmed the diagnosis subsequently by enzyme and molecular studies, which involved direct DNA sequence
\end{abstract}

Communicated by: Marinus Duran

M. Tsuji $\cdot$ H. Osaka $(\square)$

Division of Neurology, Clinical Research Institute,

Kanagawa Children's Medical Center,

2-138-4 Mutsukawa, Minami-ku,

Yokohama 232-8555, Japan

e-mail: hosaka@kcmc.jp

N. Aida

Division of Radiology, Clinical Research Institute,

Kanagawa Children's Medical Center,

2-138-4 Mutsukawa, Minami-ku,

Yokohama 232-8555, Japan

N. Furuya $\cdot$ K. Kurosawa

Division of Genetics, Clinical Research Institute,

Kanagawa Children's Medical Center,

2-138-4 Mutsukawa, Minami-ku,

Yokohama 232-8555, Japan

T. Obata $\cdot$ M. Tomiyasu

Department of Biophysics, Molecular Imaging Center,

National Institute of Radiological Sciences,

Chiba, Japan analysis and the development of a novel multiplex ligationdependent probe amplification test.

Results ${ }^{1} \mathrm{H}-\mathrm{MRS}$ analysis revealed an elevated GABA concentration in the basal ganglia $(2.9 \mathrm{mmol} / \mathrm{l})$. Based on the results of quantitative ${ }^{1} \mathrm{H}-\mathrm{MRS}$ and clinical findings, GABA-T deficiency was suspected and confirmed in cultured lymphoblasts. Molecular studies of the GABA-T gene revealed compound heterozygosity for a deletion of one exon and a missense mutation, $275 \mathrm{G}>\mathrm{A}$, which was not detected in 210 control chromosomes.

Conclusions Our results suggest that excessive prenatal GABA exposure in the central nervous system (CNS) was responsible for the clinical manifestations of GABA transaminase deficiency. Our findings suggest the dual

\author{
A. Errami \\ MRC-Holland, \\ Amsterdam, The Netherlands \\ K. M. Gibson \\ Department of Biological Sciences, \\ Michigan Technological University, \\ Houghton, MI, USA
}

G. S. Salomons · C. Jakobs

Metabolic Unit, Department of Clinical Chemistry,

VU University Medical Center,

Amsterdam, The Netherlands

H. Osaka

Molecular Pathology \& Genetics Division,

Kanagawa Cancer Center Research Institute,

Yokohama, Japan 
nature of GABA as an excitatory molecule early in life, followed by a functional switch to an inhibitory species later in development. Furthermore, quantitative ${ }^{1} \mathrm{H}-\mathrm{MRS}$ appears to be a useful, noninvasive tool for detecting inborn errors of GABA metabolism in the CNS.

\section{Abbreviations}

GABA-T Gamma aminobutyric acid transaminase

${ }^{1} \mathrm{H}-\mathrm{MRS} \quad$ Proton magnetic resonance spectroscopy

CNS Central nervous system

SSADH Succinic semialdehyde dehydrogenase

GHB 4-hydroxybutyrate

EEG Electroencephalogram

CSF Cerebrospinal fluid

DWI Diffusion-weighted image

Glx Glutamine/glutamate complex

\section{Introduction}

Disorders of gamma aminobutyric acid (GABA) metabolism are rare and manifest prominent neurological sequelae; 4aminobutyrate aminotransferase $(\gamma$-aminobutyrate: GABA transaminase, or GABA-T; OMIM 137150) deficiency is characterized by severe psychomotor retardation, hypotonia, hyperreflexia, seizures, high-pitched cry, and growth acceleration, associated with early infantile death in two siblings (one family) (Jaeken et al 1984; Jakobs et al 1993). Succinic semialdehyde dehydrogenase (SSADH) deficiency [or 4hydroxybutyric (GHB) aciduria] is the most prevalent of the GABA degradation disorders and one in which pharmacologically active GHB, as well as GABA, accumulate in patient body fluids (Jakobs et al 1993; Pearl et al 2007). Homocarnosinosis (homocarnosine is the GABA:L-histidine
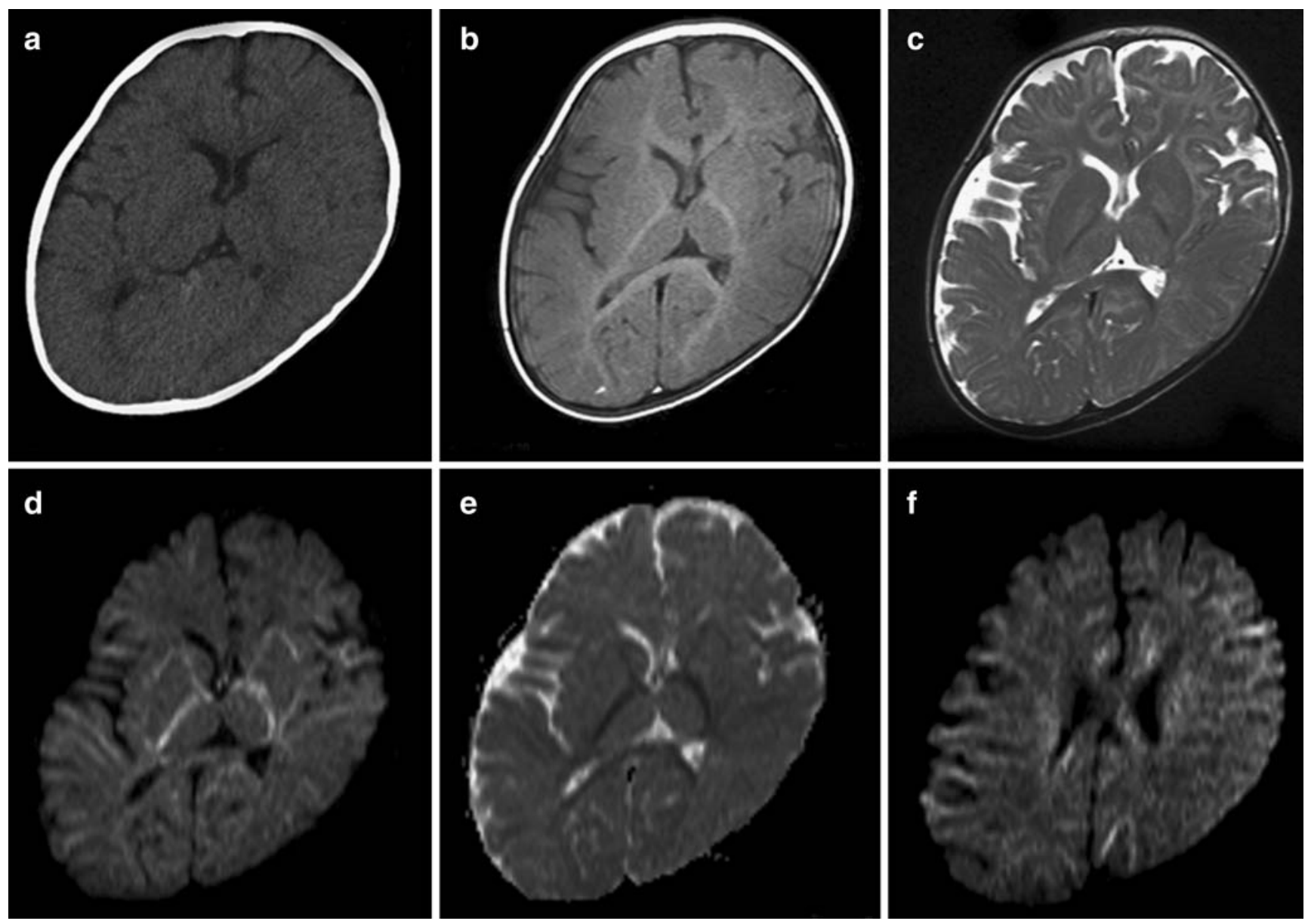

Fig. 1 Initial computed tomography (CT) and magnetic resonance imaging (MRI) findings at 8 months. Baseline CT (a), T1-weighted (b), T2-weighted (c), diffusion-weighted (d) axial MRI images, and apparent diffusion coefficients (ADC) map (e) at the level of the basal ganglia, and diffusion-weighted images (DWI) of the semioval center (f). CT (a) shows no particular abnormality, whereas T1-weighted (b) and T2-weighted (c) images suggest delayed myelination. Subcortical high white-matter signal on the T1-weighted image was not observed, and low signal on the T2-weighted image was limited to the posterior portion of the internal capsules and splenium of the corpus callosum. DWI $(\mathbf{d}, \mathbf{f})$ shows widespread high signals in the internal and external capsules and many parts of the subcortical white matter, with restricted diffusion (e) 
conjugate) is very rare (two cases) and may represent an allelic form of carnosinase deficiency(Pearl et al 2007). Considering the inhibitory nature of GABA activity in the central nervous system (CNS), the paradoxical neurological phenomenon associated with seizures in cases of GABA excess is of interest. In this study, we detected elevated GABA in a patient by proton magnetic resonance spectroscopy $\left({ }^{1} \mathrm{H}-\mathrm{MRS}\right)$ using the LCModel to quantify the spectra automatically. This method has potential application to neurological disorders such as GABA-T deficiency.

\section{Case report}

The patient was a Japanese female infant, born full term with normal delivery. She was the second child of healthy
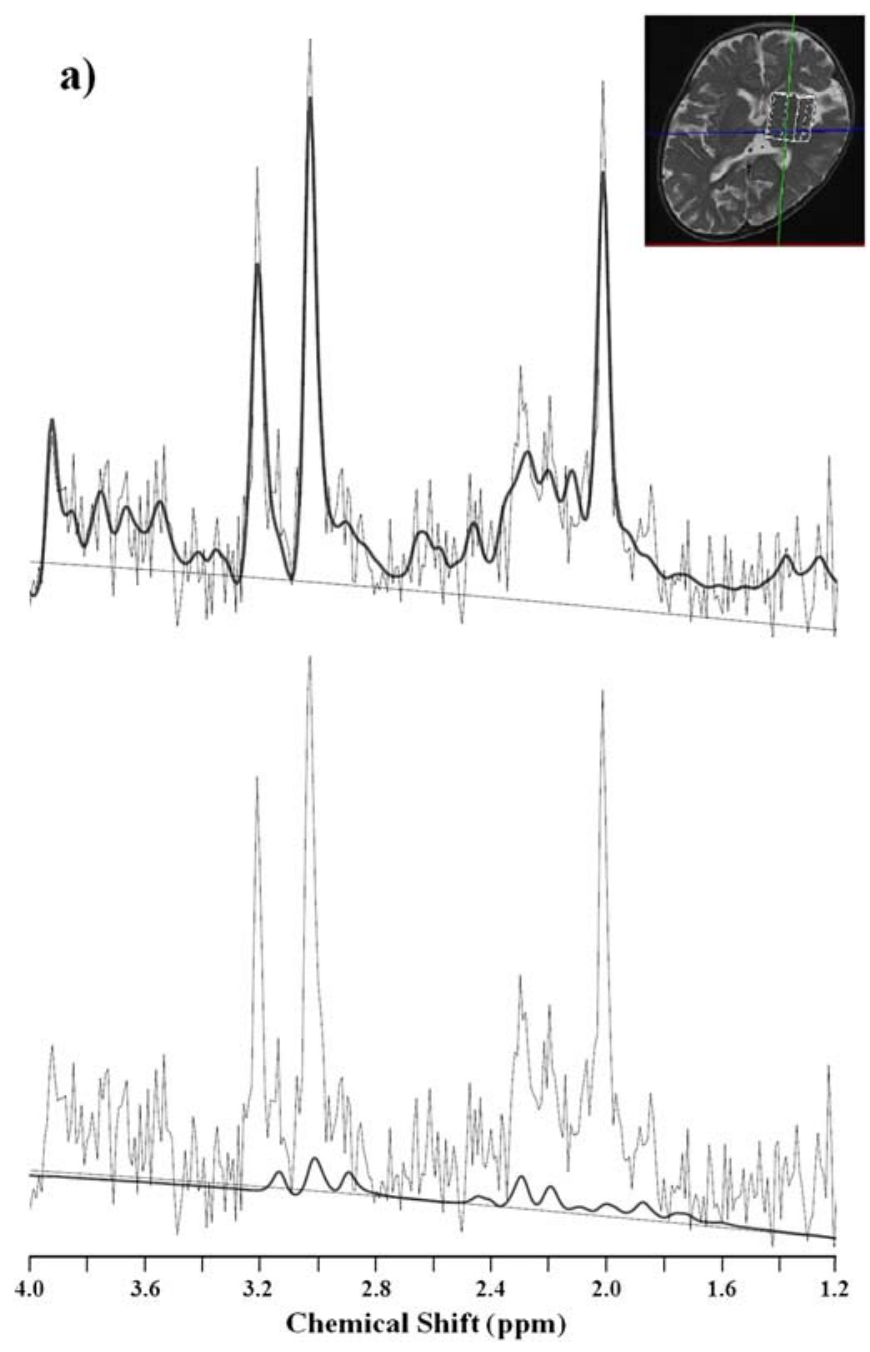

Fig. 2 LCModel outputs of in vivo proton magnetic resonance (MR) spectra from the basal ganglia (volume, 10-17.5 ml; TE/TR, 20-30/ $5000 \mathrm{~ms}$; number of excitations 6). Bold lines indicate LCModel fitting, and thin lines indicate the original spectra. Patient with gamma aminobutyric acid transaminase (GABA-T) deficiency (8 months) (a), a control individual (7 months) (b). Bold lines in the upper row are the parents. There was no consanguinity or family history of neurological disorders. A 6-year-old sister was normal. Early infancy was unremarkable. At 7 months, she was evaluated for psychomotor retardation, hypotonia, bilateral intermittent esotropia, hyperreflexia, and positive Babinski reflex. There was no dysmorphy. At age 8 months, she was admitted with decreased consciousness $48 \mathrm{~h}$ after an acute febrile illness. Respiratory distress developed that required mechanical ventilation. Steroid pulse therapy was initiated for a suspected acute encephalopathy of unknown etiology. Segmental myoclonic jerks occurred and were difficult to control, but consciousness returned. Electroencephalography (EEG) revealed diffuse slow spike and wave discharges with 1- to 2-s periods of suppression. Phenobarbital, clonazepam, valproate, and midazolam could not completely control seizures. Limb motor conduction velocities were

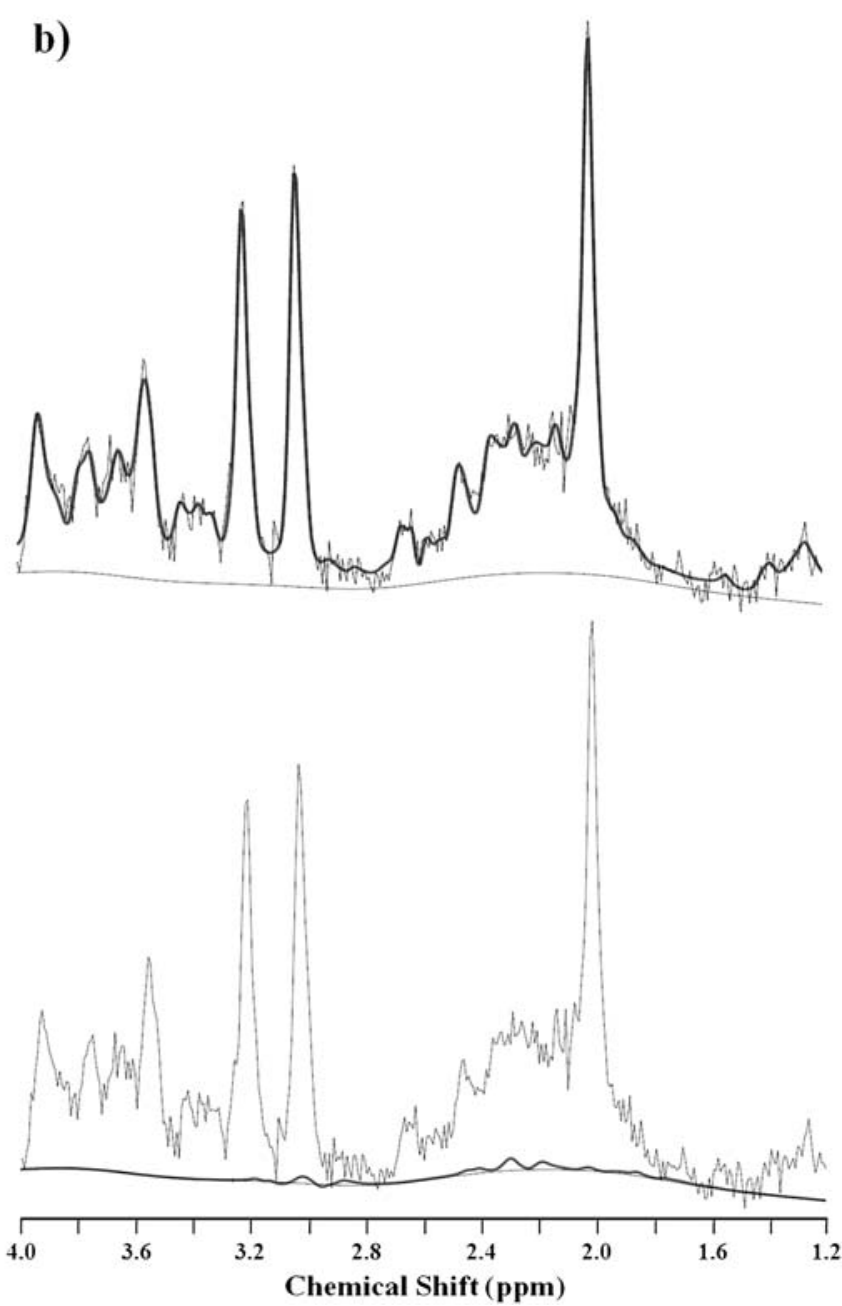

fitting curves of total spectra including all metabolites, and those in the lower row are fitting curves for GABA. The estimated absolute concentrations of GABA in patient and control are 2.9 and $0.8 \mathrm{mmol} / 1$, respectively. Normal GABA spectrum exhibits a quintet $\left({ }^{3} \mathrm{CH}_{2}\right)$ at $1.89 \mathrm{ppm}$, a triplet $\left({ }^{4} \mathrm{CH}_{2}\right)$ at $2.28 \mathrm{ppm}$, and a multiplet resembling a triplet $\left({ }^{2} \mathrm{CH}_{2}\right)$ at $3.01 \mathrm{ppm}$ (Govindaraju et al 2000) 
within normal limits. Anthropomorphic parameters revealed accelerated height in late infancy [+2.5-3.0 standard deviation (SD)], with normal head circumference and decreased weight gain. At 8 months, her height was $76 \mathrm{~cm}(+3.0 \mathrm{SD})$, weight $6745 \mathrm{~g}(-1.7 \mathrm{SD})$, and head circumference $44 \mathrm{~cm}(-0.4 \mathrm{SD})$. Nasogastric tube feeding was started due to recurrent aspiration pneumonia. At 11 months, domiciliary oxygen was introduced because of chronic respiratory failure. At the age of 28 months, her height was $96 \mathrm{~cm}(+2.9 \mathrm{SD})$, weight $10.3 \mathrm{~kg}(-1.1 \mathrm{SD})$, and head circumference $46.5 \mathrm{~cm}$ ( $-0.7 \mathrm{SD})$. Febrile illness was consistently associated with neurological deterioration, and the patient progressed to opisthotonic posturing with generalized dystonia and segmental myoclonic jerks, which never resolved while awake.

\section{Methods and results}

\section{Laboratory data}

Routine laboratory tests were normal. Amino acid analysis showed elevated free GABA in the serum and cerebral spinal fluid (CSF) at 9 months of age $(2.1 \mu \mathrm{mol} / 1$ and $1.26 \mu \mathrm{mol} / \mathrm{l}$, respectively; normal range serum $0.12-0.50$, CSF 0.04-0.12) (Jaeken et al 1984). Serum growth hormone was elevated $(8.84 \mathrm{ng} / \mathrm{ml}$; normal range 0.28 1.64). Insulin-like growth factor levels were relatively low
(54 ng/ml; normal range 37-229). An absence of GHB in urine organic acid analysis precluded SSADH deficiency as a cause of increased GABA. $\beta$-alanine and homocarnosine were not detectable on the chromatogram, and their quantitative analyses were not performed.

\section{Radiological findings}

Bone age was 1 year 8 months at the age of 1 year 10 months (TW2 method). Initial brain computed tomography (CT) was unremarkable (Fig. 1a), and brain magnetic resonance imaging (MRI) $(1.5 \mathrm{~T})$ suggested mild delay in myelination (Fig. 1b, c) without structural anomalies. Diffusion weighted images (DWI) revealed high signal intensity in the internal and external capsules and much of the subcortical white matter, with restricted apparent diffusion coefficiency (Fig. 1d-f). For quantitative ${ }^{1}$ H-MRS (age 8 months), locations were placed in the white matter (semioval center) and the basal ganglia $(10 \mathrm{ml}) .{ }^{1} \mathrm{H}-\mathrm{MR}$ spectra were obtained using the stimulated-echo acquisition mode (STEAM) sequence (Frahm et al 1987) $(\mathrm{TE} / \mathrm{TR}=$ 20/5,000 ms). To quantify the spectra, the LCModel (Provencher 1993) was used. The LCModel facilitates metabolite separation based upon differing linear combinations of spectra of individual metabolites and estimates the concentration of each metabolite concentration by comparing the proton concentration of water in identical voxels. The GABA concentration in the basal ganglia (Fig. 2) was

Table 1 Clinical, enzymatic, and molecular characteristics of gamma aminobutyric acid transaminase (GABA-T)-deficient patients

\begin{tabular}{|c|c|c|c|}
\hline Sign/symptom & Patient 1 & Patient 2 (sib of patient 1$)$ & This report \\
\hline Intractable seizures & + & + & + \\
\hline Psychomotor retardation & + & + & + \\
\hline Hypotonia & + & + & + \\
\hline High-pitched cry & + & + & - \\
\hline Hyperreflexia & + & + & + \\
\hline Lethargy & + & + & + \\
\hline Acceleration of height growth & + & + & + \\
\hline Age of death & 25 months & 12 months & Alive at 28 months \\
\hline EEG/MRI/CT abnormalities & + & + & + \\
\hline GABA-T (liver) ${ }^{\mathrm{a}}$ & $70(310-690)$ & - & - \\
\hline GABA-T (white cells) ${ }^{\mathrm{b}}$ & $1.2(20-58)$ & - & $2(23-64)$ \\
\hline Genotype $^{c}$ & c. $[659 \mathrm{G}>\mathrm{A}(+) 1433 \mathrm{~T}>\mathrm{C}]^{\mathrm{d}}$ & - & c. $[275 \mathrm{G}>\mathrm{A}]+[199-?, 316+?]$ \\
\hline Deduced effect & p.[Arg220Lys (+) Leu478Pro] & - & p.[Arg92Gln ]+[?] \\
\hline
\end{tabular}

$E E G$ electroencephalograph, MRI magnetic resonance imaging, $C T$ computed tomography, + present; - absent or not determined.

${ }^{\mathrm{a}}$ Protein $\mathrm{pmol} / \mathrm{h} / \mathrm{mg}$ (control range in parentheses). ${ }^{\mathrm{b}}$ Protein $\mathrm{pmol} / \mathrm{min} / \mathrm{mg}$ (control range in parentheses). ${ }^{\mathrm{c}}$ Reference sequence NM_000663.3; missense mutations are considered to be pathogenic, as they were not encountered in 210 control chromosomes and involve highly conserved amino acids among GABA-T species. ${ }^{\mathrm{d}}$ Following the original publication (Jaeken et al 1984), we identified the second mutation (c.1433T $>$ C) in the first described patient, confirming GABA-T deficiency at the DNA level. ${ }^{\mathrm{e}}$ In our patient, a presumed homozygous mutation was detected by direct sequence analysis; however, this was in contrast to the findings in DNA of the mother. The heterozygous mutation could not be detected in DNA of the father, therefore, a specific multiplex probe amplification test was developed. This showed the presence of a heterozygous exon deletion in DNA of the patient confirming compound heterozygosity 
significantly elevated $(2.9 \mathrm{mmol} / \mathrm{l}$; normal $1.1 \mathrm{mmol} / \mathrm{l} \pm 0.3$, $n=9$ ), but in the semioval center, GABA elevation was slight (0.8 mmol/1; normal $0.5 \mathrm{mmol} / 1 \pm 0.2, n=9)$. Glutamine/ glutamate complex (Glx) concentration was also slightly elevated in the semioval center $(11.3 \mathrm{mmol} / \mathrm{l}$ in the basal ganglia, $8.3 \mathrm{mmol} / \mathrm{l}$ in the semioval center; normal $10.1 \mathrm{mmol} / 1$ $\pm 1.5,6.6 \mathrm{mmol} / 1 \pm 1.0, n=9$, respectively). Follow-up ${ }^{1} \mathrm{H}$-MRS analysis (at 9 months of age) revealed a more pronounced GABA elevation both in the basal ganglia and in the semioval center $(5.9 \mathrm{mmol} / \mathrm{l}$ and $2.9 \mathrm{mmol} / \mathrm{l}$, respectively). Based on these data and the results of quantitative ${ }^{1} \mathrm{H}-\mathrm{MRS}$, we suspected GABA-T deficiency, which was confirmed by enzyme and molecular studies in cultured lymphoblasts (Schor et al 2001) (Table 1).

\section{Discussion}

This report is on the third patient (second family) with GABA-T deficiency and the first patient in whom ${ }^{1} \mathrm{H}-\mathrm{MRS}$ was performed. All three patients showed severe, nonspecific neurological manifestations, including psychomotor retardation, epilepsy, hypotonia, and hyperreflexia (Table 1), but our patient appeared less severely affected than the reported patients. All three also showed growth acceleration associated with increased serum growth hormone levels.

The underlying pathophysiology in GABA-T deficiency remains to be elucidated, and there is no animal model available. Evidence from animal studies indicates a neurotoxic role for supraphysiological GABA levels. For example, inhibition of GABA-T by the irreversible inhibitor, vigabatrin, induces intramyelinic edema in dogs via GABA elevation (Peyster et al 1995). Both GABA-T and SSADH deficiencies manifest seizures, which is paradoxical, as activation of the GABAergic system is predicted to be anticonvulsive. Nonetheless, it is important to remember that GABA is excitatory in the developing rodent brain and remains so for the first 1-2 weeks of life. Along these lines, the switch of GABA from a depolarizing to a hyperpolarizing response is critically important in the rodent substantia nigra pars reticulata (SNR), which has one of the highest concentrations of GABAergic neurons in the CNS (Iadarola and Gale 1982). In the murine model of SSADH deficiency, Jansen and coworkers (2008) demonstrated a significant increase in GABA in E10 embryos, which may predispose these animals to a hyperexcitatory state during development. This result, along with GABA(A) and GABA (B) receptor anomalies detected in developing SSADHdeficient mice, may reduce the seizure threshold in SSADH deficiency (Buzzi et al 2006; Wu et al 2006). Both disorders occupy juxtaposed positions in GABA degradation, and accordingly we speculate that the pathophysiological mechanisms observed in SSADH deficiency may be likely to be caused by high GABA levels, as observed in GABA-T deficiency. Neuropathology of the two index cases revealed spongy leukodystrophy, which may correspond to the white matter lesions seen on DWI in our patient. This observation may reflect changes in water motion in the axonal direction and/or axonal swelling associated with cortical neuronal damage.

Whereas ${ }^{1} \mathrm{H}-\mathrm{MRS}$ estimates in vivo neurotransmitter concentrations (Provencher 1993), quantifying GABA in nonpathological states is difficult due to interference by much larger peaks of the glutamine-glutamate complex, creatine, and large peaks of N-acetylaspartic acid (Novotny et al. 2003). However, utilizing the LCModel facilitates separation of even low-concentration species (such as GABA) from other major compounds. Screening of the metabolite concentration by ${ }^{1} \mathrm{H}$-MRS may readily reveal the pathological state, however, as in our patient. Moreover, the addition of a short exposure to ${ }^{1} \mathrm{H}-\mathrm{MRS}$ may be acceptable, even in infants and children. Increased intracranial GABA detected by ${ }^{1} \mathrm{H}-\mathrm{MRS}$ has been reported in SSADH deficiency (Ethofer et al 2004), but additional GABA-Tdeficient patients require identification in order to determine how the concentrations of intracranial GABA compare to those in the same regions of SSADH-deficient patients.

In summary, GABA transaminase deficiency represents a human model of endogenous GABA elevation, which likely occurs during critical periods of human CNS development. This disorder may offer valuable insights into the role of the GABAergic system in human brain development. Our studies further suggest that quantitative ${ }^{1} \mathrm{H}-\mathrm{MRS}$ may be clinically applicable to the inborn errors of GABA metabolism.

Acknowledgements We thank Lyuba Bayukanskaya, Ana Pop, and Rizkat Yilmaz for technical assistance. This work was supported in part by Grants-in-Aid from Scientific Research from the Ministry of Health, Labor and Welfare of Japan, Health and Labor Science Research Grant of Japan (Kokoro-Ippan-015), Takeda Science Foundation and Kanagawa Municipal Hospital Pediatric Research.

Open Access This article is distributed under the terms of the Creative Commons Attribution Noncommercial License which permits any noncommercial use, distribution, and reproduction in any medium, provided the original author(s) and source are credited.

\section{References}

Buzzi A, Wu Y, Frantseva MV et al (2006) Succinic semialdehyde dehydrogenase deficiency: GABAB receptor-mediated function. Brain Res 1090:15-22

Ethofer T, Seeger U, Klose U et al (2004) Proton MR spectroscopy in succinic semialdehyde dehydrogenase deficiency. Neurology 62:1016-1018

Frahm J, Merboldt KD, Hänicke W (1987) Localized proton spectroscopy using stimulated echoes. J Magn Reson 72:502-508 
Govindaraju V, Young K, Maudsley AA (2000) Proton NMR chemical shifts and coupling constants for brain metabolites. NMR Biomed 13:129-153

Iadarola MJ, Gale K (1982) Substantia nigra: site of anticonvulsant activity mediated by gamma-aminobutyric acid. Science 218:12371240

Jaeken J, Casaer P, de Cock P et al (1984) Gamma-aminobutyric acidtransaminase deficiency: a newly recognized inborn error of neurotransmitter metabolism. Neuropediatrics 15:165-169

Jakobs C, Jaeken J, Gibson KM (1993) Inherited disorders of GABA metabolism. J Inherit Metab Dis 16:704-715

Jansen EE, Struys E, Jakobs C, Hager E, Snead OC 3rd, Gibson KM (2008) Neurotransmitter alterations in embryonic succinate semialdehyde dehydrogenase (SSADH) deficiency suggest a heightened excitatory state during development. BMC Dev Biol 8:112

Novotny EJ Jr, Fulbright RK, Pearl PL, Gibson KM, Rothman DL (2003) Magnetic resonance spectroscopy of neurotransmitters in human brain. Ann Neurol 54(Suppl 6):S25-S31
Pearl P, Jakobs C, Gibson K (2007) Disorders of $\beta$ - and $\gamma$-amino acids in free and peptide-linked forms. In The metabolic and molecular bases of inherited disease Part 8, amino acids, Ch 91. McGrawHill, New York

Peyster RG, Sussman NM, Hershey BL et al (1995) Use of ex vivo magnetic resonance imaging to detect onset of vigabatrin-induced intramyelinic edema in canine brain. Epilepsia 36:93-100

Provencher SW (1993) Estimation of metabolite concentrations from localized in vivo proton NMR spectra. Magn Reson Med 30:672-679

Schor DS, Struys EA, Hogema BM, Gibson KM, Jakobs C (2001) Development of a stable-isotope dilution assay for gammaaminobutyric acid (GABA) transaminase in isolated leukocytes and evidence that GABA and beta-alanine transaminases are identical. Clin Chem 47:525-531

Wu Y, Buzzi A, Frantseva M et al (2006) Status epilepticus in mice deficient for succinate semialdehyde dehydrogenase: GABAA receptor-mediated mechanisms. Ann Neurol 59:42-52 